\title{
INDEX OF VOLUME 45
}

\section{AUTHORS}

Adams, C. R., and Morse, A. P. Random sampling in the evaluation of a Lebesgue integral, 442.

Agnew, R. P. Properties of generalized definitions of limit, 689.

- See Reviews, under Bowman.

Archibald, R. C. History of the American Mathematical Society, 1888-1938, 31.

Aucoin, A. A., and Parker, W. V. Diophantine equations whose members are homogeneous, 330.

Ayres, W. L. Reports of meetings of the American Mathematical Society: Semicentennial Celebration, 1; November meeting in Cleveland, 193; April meeting in Chicago, 492; September meeting in Madison, 801.

Baten, W. D. See Reviews, under Hostelet.

Bell, E. T. A duality for certain difference equations, 145.

See Reviews, under Hardy.

Boas, R. P. The Stieltjes moment problem for functions of bounded variation, 399.

Bourgin, D. G., and Duffin, R. The Dirichlet problem for the vibrating string equation, 851.

The Laplace Heaviside method for boundary value problems, 859 .

Brauer, A. On addition chains, 736.

Buchanan, H. E. See Reviews, under Smart.

Cairns, S. S. See Reviews, under Weil.

Campbell, A. D. See Reviews, under Levinson.

Carpenter, A. F. Involutory systems of curves on ruled surfaces, 107.

Carslaw, H. S., and Jaeger, J. C. On Green's functions in the theory of heat conduction, 407.

Carroll-Rusk, E. See Snyder, V.

Chernick, J. On Fermat's simple theorem, 269.

Church, A. See Reviews, under Carnap.

Coble, A. B. Cremona transformations with an invariant rational sextic, 285.

Coburn, N. $V_{m}$ in $S_{n}$ with planar points $(m \geqq 3), 774$.

Court, N. A. See Reviews, under Campbell.

Curry, H. B. A note on the reduction of Gentzen's calculus LJ, 288.

Cutler, E. H. See Reviews, under Bückner.

Day, M. M. Regularity of function-to-function transformations, 296.

DeCicco, J. J. The analogue of the Moebius group of circular transformations in the Kasner plane, 936.

Derry, D. On finite abelian p-groups, 874.

Dickson, L. E. All integers except 23 and 239 are sums of eight cubes, 588.

Dodd, E. L. See Reviews, under von Mises.

Doob, J. L. See Reviews, under Borel, Ville.

Dresden, A. See Reviews, under Semicentennial Addresses.

Duffin, R. See Bourgin, D. G.

Dwyer, W. A. Certain incomplete numerical functions, 101.

Eaton, J. E. A formula for the coefficients of the cyclotomic polynomial, 178.

Eckart, C., and Young, G. A principal axis transformation for non-hermitian matrices, 118.

Emch, A. New point configurations and algebraic curves connected with them, 731. 
Engstrom, H. T. On fundamental systems of symmetric functions, 404.

Everett, C. J. Rings as groups with operators, 274.

Fialkow, A. Totally geodesic Einstein spaces, 423.

Ficken, F. A. See Reviews, under Reports of a Mathematical Colloquium.

Flexner, W. See Reviews, under Slichter.

Fort, T. The Euler-Maclaurin summation formula, 748.

See Reviews, under Archibald.

Franklin, P. See Reviews, under Karelitz.

Garabedian, H. L. A sufficient condition for Cesdro summability, 592.

- Theorems associated with the Riesz and the Dirichlet's series methods of summation, 891.

Gentry, F. C. Cremona involutions determined by a pencil of surfaces, 614.

Gill, B. P. See Reviews, under Weber.

Graustein, W. C. See Reviews, under Schouten.

Greenwood, J. A. See Reviews, under Fréchet.

Greville, T. N. E. See Reviews, under Boehm.

Grove, V. G. $A$ tensor analysis for $a V_{k}$ in a projective space $S_{n}, 385$.

Hailperin, T. On contiguous point spaces, 172.

Hall, D. W. An example in the theory of pointwise periodic homeomorphisms, 882.

Hall, D. W., and Wallace, A. D. Some invariants under monotone transformations, 294.

Hamilton, O. H. Concerning continua in a separable space which do not cross, 114.

Hedlund, G. A. The dynamics of geodesic flows, 241.

Hestenes, M. R., and Reid, W. T. A note on the Weierstrass condition in the calculus of variations, 471.

Hille, E. See Reviews, under Davis, Nevanlinna, Singh, Vitali.

Hollcroft, T. R. The maximum number of distinct contacts of two algebraic surfaces, 158.

- Reports of meetings of the American Mathematical Society: Semicentennial Celebration, 1; October meeting in New York, 47; Annual meeting of the Society in Richmond and Williamsburg, 197; February meeting in New York, 337; April meeting in Durham, 487.

Hopkins, C. See Reviews, under Schwerdtfeger.

Ingraham, M. H. Report of the Semicentennial Celebration, 1.

Jacobson, N. An application of E. H. Moore's determinant of a hermitian matrix, 745.

Jaeger, J. C. See Carslaw, H. S.

Jones, B. W. See Reviews, under Dickson.

Jones, F. B. Concerning the boundary of a complementary domain of a continuous curve, 428.

Concerning certain linear abstract spaces and simple continuous curves, 623.

Kempner, A. J. See Reviews, under Landau.

Kline, J. R. See Reviews, under Bouligand.

Kline, M. Representation of homeomorphisms in Hilbert space, 138.

Kolchin, E. R. On the basis theorem for infinite systems of differential polynomials, 923.

- See Ritt, J. F.

Kroon, R. P. See Reviews, under Kowalewski.

Kuhn, H. W. See Reviews, under Miller.

Lamson, K. W. See Reviews, under Rojansky.

Latshaw, V. V. See Reviews, under Valentiner.

Lehmer, D. H. A factorization theorem applied to a test for primality, 132.

Leighton, W. A test-ratio test for continued fractions, 97.

Leighton, W., and Scott, W. T. A general continued fraction expansion, 596. 
Lengyel, B. A. Bounded self-adjoint operators and the problem of moments, 303.

Levi, H. On the values assumed by polynomials, 570.

Levine, J. Metric spaces with geodesic Ricci curves. II, 122.

Groups of motions in conformally flat spaces. II, 766.

Lewis, D. C. See Reviews, under Frazer.

Lorch, E. R. Bicontinuous linear transformations in certain vector spaces, 564 .

Means of iterated transformations in reflexive vector spaces, 945.

Lowan, A. N. On Green's functions in the theory of heat conduction in spherical coordinates, 310.

- On wave motion in an infinite solid bounded internally by a cylinder or a sphere, 316.

- On the computation of the second differences of the $\operatorname{Si}(x), \operatorname{Ei}(x)$ and $\mathrm{Ci}(x)$ functions, 583.

- Corrections to "On Green's functions in the theory of heat conduction in spherical coordinates," 951.

McCoy, N. H. Generalized regular rings, 175.

- Concerning matrices with elements in a commutative ring, 280.

- A theorem on matrices over a commutative ring, 740.

$\mathrm{McEwen,} \mathrm{W.} \mathrm{H.} \mathrm{On} \mathrm{the} \mathrm{simultaneous} \mathrm{approximation} \mathrm{of} \mathrm{a} \mathrm{function} \mathrm{and} \mathrm{its} \mathrm{derivatives} \mathrm{by}$ sums of Birkhoff type, 576.

McKinsey, J. C. C. A note on Reichenbach's axioms for probability implication, 799.

McShane, E. J. On the uniqueness of the solutions of differential equations, 755.

MacDonald, J. K. L. On bounds for parameters in n-noded solutions of Sturm-Liouville equations, 164.

- See Reviews, under Contributions to the Mechanics of Solids.

MacLane, S. The universality of formal power series fields, 888 .

MacNeille, H. M. Extension of a distributive lattice to a Boolean ring, 452.

See Reviews, under Foradori.

Manning, W. A. On transitive groups that contain certain transitive subgroups, 783.

Maximoff, I. On approximately continuous functions, 264.

Michal, A. D. General differential geometries and related topics, 529.

Morse, A. P. See Adams, C. R.

O'Connor, R. E. Quadratic and linear congruence, 792.

Ogburn, J. H. See Reviews, under Deming.

Oldenburger, R. Decomposition of elements in abelian groups, 152.

Opatowski, I. Combinatoric interpretation of a formula for the nth derivative of a function of a function, 944.

Ore, O. James Pierpont-in memoriam, 481.

Parker, W. V. See Aucoin, A. A.

Pearl, R. See Reviews, under Rashevsky.

Perkins, F. W. See Reviews, under Vasilesco.

Putnam, T. M. Derrick Norman Lehmer-in memoriam, 209.

- Reports of meetings of the American Mathematical Society: Semicentennial Celebration, 1; November meeting in Los Angeles, 195; April meeting at Stanford University, 497.

Rainich, G. Y. See Reviews, under Shabde.

Randolph, J. F. See Reviews, under Riemann.

Raynor, G. E. On Serret's integral formula, 911.

Reid, W. T. Some remarks on linear differential systems, 414. See Hestenes, M. R. 
Reynolds, J. B. See Reviews, under Coe.

Richardson, R. G. D. Report of the Semicentennial Celebration, 1.

The Society establishes a new periodical, 641 .

Ritt, J. F., and Kolchin, E. R. On certain ideals of differential polynomials, 895.

Robinson, R. M. On numerical bounds in Schottky's theorem, 907.

Rosenthall, E. Representation of numbers in ternary quadratic forms, 261.

Ross, A. E. A theorem on simultaneous representation of primes and its corollaries, 899.

Rosser, B. On the first case of Fermat's last theorem, 636.

- See Reviews, under Gentzen, Lautman.

Rothe, E. Topological proofs of uniqueness theorems in the theory of differential and integral equations, 606.

Scarborough, J. B. See Reviews, under Stephens.

Schilling, O. F. G. See Reviews, under Lie.

Schlesinger, F. Ernest William Brown-in memoriam, 343.

Scott, W. T. See Leighton, W.

Sewell, W. E. Jackson summation of the Faber development, 187.

Shook, C. A. See Reviews, under Pérès.

Shreve, D. R. On a certain class of symmetric hypersurfaces, 948.

Sinkov, A. A note on a paper by J. A. Todd, 762.

Smail, L. L. See Reviews, under Moore.

Smith, F. C. On the logarithmic solutions of the generalized hypergeometric equation when $p=q+1,629$.

- Relations among the fundamental solutions of the generalized hypergeometric equation when $p=q+1$. II. Logarithmic cases, 927.

Smith, P. A. See Reviews, under Newman.

Snyder, V. See Reviews, under Prüfer, Room.

Snyder, V., and Carroll-Rusk, E. A Cremona involution in $S_{3}$ without a surface of invariant points, 141.

Spencer, H. E. See Reviews, under Appell.

Steenrod, N. E. See Reviews, under Reidemeister.

Stoll, R. R. Fundamental regions for the simple group of order 60 in $S_{4}, 326$.

Stone, M. H. See Reviews, under Julia.

Stopher, E. C. Point set operators and their interrelations, 758.

Struik, D. J. See Reviews, under Jeffreys.

Synge, J. L. See Reviews, under Blaschke.

Szegö, G. Concerning sets of polynomials orthogonal simultaneously on several circles, 129.

Thomas, T. Y. On the singular point locus in the theory of fields of parallel vectors, 436 . Imbedding theorems in differential geometry, 841.

Thurston, H. S. On the number of sets conjugate to a matrix with linear elementary divisors, 474.

Trjitzinsky, W. J. See Reviews, under Buhl, Kryloff.

Vanderslice, J. L. See Reviews, under Weatherburn.

Vickery, C. W. Spaces of uncountably many dimensions, 456.

Walker, R. J. See Reviews, under Blaschke.

Wallace, A. D. On non-boundary sets, 420. See Hall, D. W.

Walsh, J. L. Note on the location of zeros of the derivative of a rational function whose zeros and poles are symmetric in a circle, 462.

Ward, M. A note on divisibility sequences, 334 . 
A characterization of Dedekind structures, 448.

Warnock, W. G. Triple systems as ruled quadrics, 476.

Webster, M. S. Note on certain Lagrange interpolation polynomials, 870.

Wernick, W. An enumeration of logical functions, 885.

Williamson, J. On the determinant of an automorph of a non-singular skew-symmetric matrix, 307.

- Note on a principal axis transformation for non-hermitian matrices, 920.

Wilson, R. A note on the asymptotic properties of orthogonal polynomials, 190.

Yeaton, C. H. See Reviews, under Appell.

Young, G. See Eckart, C.

Zuckerman, H. S. The computation of the smaller coefficients of $J(\tau), 917$. 


\section{REVIEWS}

Appell, P. Analyse Mathêmatique. Vol. 1. Analyse des Courbes, Surfaces et Fonctions Usuelles. Intégrales Simples. 5th edition. C. H. Yeaton, 219.

— Analyse Mathématique. Vol. 2. Équations Différentielles. Développements en Séries. Nombres Complexes. Intégrales Multiples. 5th edition. H. E. Spencer, 653.

Archibald, R. C. A Semicentennial History of the American Mathematical Society, 1888-1938. (American Mathematical Society Semicentennial Publications, vol. 1.) T. Fort, 50.

Blaschke, W. Ebene Kinematik. (Hamburger mathematische Einzelschriften, no. 25.) J. L. Synge, 814 .

Blaschke, W., and Bol, G. Geometrie der Gewebe. R. J. Walker, 652.

Boehm, C., Rose, E., and Lorenz, P. Versicherungsmathematische Aufgabensammlung. Vol. 1. Beiträge und Deckungsriicklagen in der Lebensversicherung. Vol. 2. Umwandlung von Lebensversicherungen. T. N. E. Greville, 504.

Bogoliuboff, N. See Kryloff, N.

Bol, G. See Blaschke, W.

Borel, E. Valeur Pratique et Philosophie des Probabilités. (Traité du Calcul des Probabilités et de ses Applications, vol. 4, no. 3.) J. L. Doob, 651.

Bouligand, G. Les Definitions Modernes de la Dimension. J. R. Kline, 503.

Bowman, F. Introduction to Bessel Functions. R. P. Agnew, 346.

Bücker, H. Zur Differentialgeometrie der Kurven und Flächen fester Breite. (Schriften der Königsberger gelehrten Gesellschaft, vol. 14, no. 1.) E. H. Cutler, 217.

Buhl, A. Nouveaux Éléments d'Analyse. Calcul Infinitésmal. Gêométrie. Physique Thêorique. Vol. 2. Variables Complexes. W. J: Trjitzinsky, 60.

Campbell, A. D. Advanced Analytic Geometry. N. A. Court, 347.

Carnap, R. Foundations of Logic and Mathematics. (International Encyclopedia of Unified Science, vol. 1, no. 3.) A. Church, 821.

Coe, C. J. Theoretical Mechanics. A Vectorial Treatment. J. B. Reynolds, 346.

Collar, A. R. See Frazer, R. A.

Contributions to the Mechanics of Solids. (Dedicated to S. Timoshenko.) J. K. L. MacDonald, 821.

Davis, H. T. The Theory of Linear Operators from the Standpoint of Differential Equations of Infinite Order. E. Hille, 220.

Deming, W. E. Some Notes on Least Squares. J. H. Ogburn, 502.

Dickson, L. E. New First Course-in the Theory of Equations. B. W. Jones, 820.

Duncan, W. J. See Frazer, R. A.

Foradori, E. Grundgedanken der Teiltheorie. H. M. MacNeille, 62.

Frazer, R. A., Duncan, W. J., and Collar, A. R. Elementary Matrices and some Applications to Dynamics and Differential Equations. D. C. Lewis, 825.

Fréchet, M. Méthode des Fonctions Arbitraires. Thêorie des Événements en Châ̂ne dans le Cas d'un Nombre Fini d'États Possibles. (Traité du Calcul des Probabilités et de ses Applications, vol. 1, no. 3.) J. A. Greenwood, 56.

Garrelts, J. M. See Karelitz, G. B.

Gentzen, G. Die Gegenwärtige Lage in der mathematischen Grundlagenforschung. Neue Fassung des Wiederspruchsfreiheitsbeweises für die reine Zahlentheorie. (Forschungen zur Logik und zur Grundlegung der exakten Wissenschaften, new series, no. 4.) B. Rosser, 812.

Hardy, G. H., and Wright, E. M. An Introduction to the Theory of Numbers. E. T. Bell, 507. 
Hostelet, G. Les Fondements Expérimentaux de l'Analyse Mathématique des Faits Statistiques. (Actualités Scientifiques et Industrielles, no. 552.) W. D. Baten, 345.

—Le Concours de l'Analyse Mathématique à l'Analyse Expérimentale des Faits Statistiques. (Actualités Scientifiques et Industrielles, no. 585.) W. D. Baten, 509.

Jeffreys, H. Scientific Interference. D. J. Struik, 213.

Julia, G. Introduction Mathématique aux Thêories Quantiques. Part 2. (Cahiers Scientifiques, vol. 19.) M. H. Stone, 59.

Karelitz, G. B., Ormondroyd, J., and Garrelts, J. M. Problems in Mechanics. P. Franklin, 824.

Kowalewski, G. Grundbegriffe und Hauptsätze der höheren Mathematik, insbesondere für Ingenieure und Naturforscher. R. P. Kroon, 505.

Kryloff, N., and Bogoliuboff, N. Introduction à la Mécanique Non-Linéaire. (In Russian.) W. J. Trjitzinsky, 62.

Landau, E. Über einige neurere Fortschritte der additiven Zahlentheorie. (Cambridge Tracts in Mathematics and Mathematical Physics, no. 35.) A. J. Kempner, 216.

Lautman, A. Essai sur l'Unité des Sciences Mathématiques dans leur Développement Actuel. (Actualités Scientifiques et Industrielles, no. 589.) B. Rosser, 511.

Levinson, H. C. Your Chance to Win. The Laws of Chance and Probability. A. D. Campbell, 506.

Lie, S. Gesammelte Abhandlungen. Geometrische Abhandlungen. Vols. 1 and 2. O. F. G. Schilling, 513.

Lorenz, P. See Boehm, C.

Miller, G. A. The Collected Works of George Abram Miller. Vol. 2. H. W. Kuhn, 644.

von Mises, R. Probability, Statistics, and Truth. Translated by J. Neyman, D. Scholl, and E. Rabinowitsch. E. L. Dodd, 815.

Moore, C. N. Summable Series and Convergence Factors. (American. Mathematical Society Colloquium Publications, vol. 22.) L. L. Smail, 218.

Nevanlinna, R. Eindeutige analytische Funktionen. (Grundlehren der mathematischen Wissenschaften, vol. 46.) E. Hille, 52.

Newman, M. H. A. Elements of the Topology of Plane Sets of Points. P. A. Smith, 822.

Neyman, J. See von Mises, R.

Ormondroyd, J. See Karelitz, G. B.

Pérès, J. Mécanique des Fluides. C. A. Shook, 512.

Prüfer, H. Projektive Geometrie. V. Snyder, 820.

Rabinowitsch, E. See von Mises, R.

Rashevsky, N. Mathematical Biophysics. Physicomathematical Foundations of Biology. R. Pearl, 223.

Reidemeister, K. Topologie der Polyeder und kombinatorische Topologie der Komplexe. (Mathematik und ihre Anwendungen, vol. 17.) N. E. Steenrod, 224.

Reports of a Mathematical Colloquium. Series 2, no. 1. F. A. Ficken, 813.

Riemann, B. Partielle Differentialgleichungen und ihre Anwendungen auf physikalische Fragen. J. F. Randolph, 652.

Rojansky, V. Introductory Quantum Mechanics. K. W. Lamson, 348.

Room, T. G. The Geometry of Determinantal Loci. V. Snyder, 499.

Rose, E. See Boehm, C.

Sansone, G. See Vitali, G.

Scholl, D. See von Mises, R.

Schouten, J. A., and Struik, D. J. Einführung in die neuren Methoden der Differentialgeometrie. Vol. 1. Algebra und Übertragungslehre. Vol. 2. Geometrie. 2d edition. W. C. Graustein, 649. 
Schwerdtfeger, H. Les Fonctions de Matrices. I. Les Fonctions Univalentes. (Actualités Scientifiques et Industrielles, no. 649.) C. Hopkins, 818.

Semicentennial Addresses. (American Mathematical Society Semicentennial Publications, vol. 2.) A. Dresden, 50.

Shabde, N. G. The General Field Theory of Schouten and Van Dantzig. (Lucknow University Studies.) G. Y. Rainich, 224.

Singh, A. N. The Theory and Construction of Nondifferentiable Functions. (Lucknow University Studies.) E. Hille, 217.

Slichter, C. S. Science in a Tavern. W. Flexner, 510.

Smart, W. M. Stellar Dynamics. H. E. Buchanan, 504.

Stephens, E. Elementary Theory of Operational Mathematics. J. B. Scarborough, 506.

Struik, D. J. See Schouten, J. A.

Timoshenko, S. See Contributions to the Mechanics of Solids.

Valentiner, S. Vectoranalysis. V. V. Latshaw, 505.

Vasilesco, F. La Notion de Point Irrégulier dans le Problème de Dirichlet. Actualités Scientifiques et Industrielles, no. 660.) F. W. Perkins, 817.

Ville, J. Étude Critique de la Notion de Collectif. (Monographies des Probabilités, no. 3.) J. L. Doob, 824.

Vitali, G., and Sansone, G. Moderna Teoria delle Funzioni di Variabile Reale. Part 2. Sviluppi in Serie di Funzioni Ortogonali. (Monografie di Matematica Applicata per cura del Consiglio Nazionale delle Ricerche.) E. Hille, 218.

Weatherburn, C. E. An Introduction to Riemannian Geometry and the Tensor Calculus. J. L. Vanderslice, 222.

Weber, W. Die Pellsche Gleichung. (Deutsche Mathematik, supplement no. 1.) B. P. Gill, 819.

Weil, A. Sur les Espaces à Structure Uniforme et sur la Topologie Générale. S. S. Cairns, 59.

Wright, E. M. See Hardy, G. H.

Abstracts of Papers Presented to the Society, 67, 228, 355, 517, 660, 829.

New Publications, 94, 239, 382, 525, 687, 840.

Notes, $63,225,349,514,655,826$. 


\section{NOTES AND OTHER ITEMS}

Academies, Associations, Congresses, and Societies:

American Mathematical Society: Annual Meeting (1938), 197; Annual Meeting (1940), 339, 488; Auditors' Report, 202; Bôcher Prize, 199; Budget, 202; Bulletin, 805; By-Laws, 203, 805; Cole Prize in Algebra, 203; Colloquium Lectures, 802, 805; Colloquium Publications, 805, 826; Committee on Abstract Journal, 203, 339; on E. W. Brown Bequest, 338; on Cole Prize in Algebra, 488; on the International Congress (1940), 47, 203; on Methods of Nominating and Electing Council Members, 339, 804; on Nominations, 201, 339; on Places of Meetings, 339; on Reorganization of the Business of the Secretariat, 805; to Select Hour Speakers, 201; on Semicentennial, 2, 3, 203; on Special Funds, 339; Dates and Places of Meetings, 203, 339, 488, 804; Election of Officers, 202; Gibbs Lecture (1939), 203, 339; Invitations for Meetings, 202, 203, 339; Librarian's Report, 202; Mathematical Reviews, 641, 804; New Members Admitted, 200, 338, 488, 803; Representatives on the Council of the American Association for the Advancement of Science, 804; on the Board of the American Year Book, 805; at the Celebration of the Fiftieth Anniversary of the Founding of the Catholic University of America, 804; at the Installation of the Director of the Cooper Union for the Advancement of Science and Art, 201; at the Centennial Celebration of Duke University, 338; at the Sesquicentennial Celebration of Georgetown University, 804; at the Inauguration of the President of Kent State University, 201; at the Inauguration of the President of Mount Union College, 201; at the Celebration of the Fiftieth Anniversary of the Founding of the University of New Mexico, 804; at the Inauguration of the President of Tufts College, 201; Semicentennial Celebration, 1; Semicentennial Publications, 6; Statistics, 1, 2, 201, 802; Summer Meeting, 1; Transactions, 63; Treasurer's Report, 202.

Associations for the Advancement of Science: American, 197, 198, 201, 804; French, 514.

Academy of Sciences, Paris, 225; Actuarial Society of America, 22; American Academy of Arts and Sciences, 22, 23; American Astronomical Society, 22; American Institute of Actuaries, 22; American Institute of Electrical Engineers, 22, 826; American Philosophical Society, 22, 23, 515; American Physical Society, 22, 24; American Society of Civil Engineers, 22; American Society of Mechanical Engineers, 22, 24; Amsterdam Mathematical Society, 4, 22; Association for Symbolic Logic, 22; Astronomical Society of the Pacific, 349; Henri Becquerel Foundation, 225; Cambridge Philosophical Society, 24; Carnegie Corporation, 642; College Entrance Examination Board, 8; Cowles Commission, 225; Danish Mathematical Society, 24; Deutsche Mathematiker Vereinigung, 22, 25; Econometric Society, 22; Edinburgh Mathematical Society, 22; Eötvös Loránd Society of Mathematics and Physics, 25; Girbal-Baral Foundation, 225; Guggenheim Memorial Foundation, 22; Institute for Advanced Study, 828; Institute of Mathematical Statistics, 22; International Congress of Mathematicans, 2, 804; London Mathematical Society, 7, 17, 22; Mathematical Association of America, 4, 6, 22, 25, 197, 198, 199, 226, 514; National Academy of Sciences, 22; National Council of Teachers of Mathematics, 22, 26, 199; National Research Council, 22, 26, 201, 514, 656; National Research Council (Canada), 22; University of Notre Dame, 655; Sixth Pacific Science Congress, 514; Polish Mathematical Society, 22, 27; Research Corporation of New York, 349; Rockefeller Foundation, 642; 
Royal Academy of Belgium, 514; Royal Dutch Academy of Sciences, 655; Royal Society, 63, 225, 226; Royal Society of Edinburgh, 349; Russian Society of Philosophy of Science in Paris, 22, 27; Schweizerische Mathematische Gesellschaft, 22, 28; Sociedad Matemática Española, 28; Société Mathématique de France, 22, 28; Société Mathématique de Grèce, 22, 28; Société Physico-Mathématique à l'Université de Kazan, 26; Society for the Promotion of Engineering Education, 22; Unione Matematica Italiana, 22, 29; Works Progress Administration, 350 .

Books, Announcement of New, 6, 826 .

Doctorates, American, 350.

Journals:

Bulletin of the American Mathematical Society, 805; Mathematical Reviews, 641, 804; Reports of a Mathematical Colloquium, 514; Transactions of the American Mathematical Society, 63.

National Research Fellowships, 656.

Papers and Communications Presented to the Society, Authors:*

Adkisson, V. W., 206 (44; 786), 496 (355), (831); Agnew, R. P., 807 (661), 811 (661), (831); Albert, A. A., 207 (67); Albert, G. E., 194 (44; 639); Allendoerfer, C. B., 206 (67), (831); Ambrose, W., 811 (661), (833); Arnold, H. A., 196 (44; 755), 497 (355), 806 (661), (832); Aroian, L. A., (832); Aucoin, A. A., 495 (355); Ayres, W. L., 807 (662).

Baer, R., 194 (44; 780), 195 (44; 779), 195 (44; 779$), 489$ (356), 806 (662), 808 (662), 810 (662); Barnett, I. A., 494 (356); Barone, H. G., 490 (356); Bartky, W., 494 (356); Basoco, M. A., 194 (44; 780); Beckenbach, E. F., 207 (85), 494 (357); Becker, M. F., (662); Bell, C., 196 (44; 775), 498 (357); Bell, P. O., 806 (663); Bergmann, S., 807 (663); Bernstein, B. A., 497 (357); Betz, E. E., 490 (517); Birkhoff, G., 340 (86), 341 (86), 808 (357), 808 (517), 809 (663); Blackall, C. J., 204 (67); Blau, J. H., 339 (228); Blumenthal, L. M., 204 (68), 496 (357), (832); Boas, R. P., 207 (68), 207 (68), 809 (517); Bochner, S., 207 (68), (832); Bohr, H., 205 (86); Bower, O. K., 341 (86), 341 (87); Brauer, A., (833); Brauer, R., 489 (358), 808 (663); Brinkmann, H. W., 48 (44; 772); Bristow, L., 48 (44; 785); Brown, O. E., 194 (44; 780); Burington, R. S., 194 (44; 780), 811 (664).

Cairns, S. S., 340 (228), (664); Calkin, J. W., 207 (68); Cameron, R. H., 204 (69), 489 (358), 809 (517); Camp, C. C., 495 (358); Campaigne, H. H., 206 (69), 494 (358), 807 (664); Cassity, C. R., 490 (518); Church, A., 490 (234); Churchill, R. V., 494 (359); Cohen, L. W., 206 (69), 490 (359); Comenetz, G., 341 (87); Coral, M., 194 (44; 781); Courant, R., 48 (44; 772$), 48$ (44; 772), 206 (70); Coxeter, H. S. M., 806 (664); Craig, H. V., 194 (44; 781); Cramér, H., 342 (359).

Darkow, M. D., 207 (44; 786); Davis, R., 811 (674); DeCicco, J. J., 48 (44; 640), 195 (44; 781), 340 (87), 340 (230), 341 (87), 491 (365), 809 (518), 809 (518), 810 (669), (829), (830); Derry, D., 494 (234); Dilworth, R. P., 196 (44; 775), 342 (92), 495 (92), 809 (518), 809 (519), (833), (833); Dimsdale, B., 806 (665); Dines, L. L., 805 (675); Dobbie, J. M., 194 (44; 782); Doob, J. L., 494 (359), (833); Douglas, J., 48 (44; 640), 48 (44; 640), 48 (44; 640), 48 (44; 785), 207 (44; 787); Dressel, F. G., 490 (360); Dribin, D. M., 493 (360); Duffin, R. J., 493 (234), 808

* Numbers within parentheses refer to abstracts, and those outside parentheses refer to titles as listed in reports of meetings. 
(680); Dunford, N., 206 (88); Dushnik, B., 811 (829); Duthie, W. D., 48 (44; 640); Dye, L. A., 204 (70), 489 (360); Dyer-Bennet, J., 808 (357).

Eaton, J. E., 340 (228), 809 (520), (665); Engstrom, H. T., 342 (235); Epstein, B., 808 (665).

Favila, R. A., 497 (360); Feld, J. M., 340 (229), 490 (361); Fialkow, A., (834); Ficken, F. A., 48 (44; 772$)$, 811 (665); Flanders, D. A., 205 (86); Flexner, W. W., 207 (70); Ford, L. R., 205 (70); Fort, T., 48 (44; 773); Foster, M. C., (834); Friedman, B., 806 (666); Friedrichs, K. O., 205 (70).

Galbraith, A. S., 489 (361); Garabedian, H. L., 207 (88), (834); Gatewood, B. E., 806 (666); Gelbart, A., (834); Gentry, F. C., 341 (229); George, T. S., (835); Gilbert, P. W., (835); Givens, W., 194 (44; 782); Gödel, K., 204 (93); Goldberg, M., (666); Goldstine, H. H., 494 (361), 496 (363); Gore, G. D., 495 (361); Gorn, S., 207 (71), 207 (71); Graves, L. M., 494 (235), 810 (666), 810 (667); Greville, T. N. E., 489 (362).

Hall, D.W., 48 (44; 773), 196 (44; 776), 204 (88), 207 (71), 490 (519), 807 (829), 808 (667), 810 (667); Hall, M., 48 (44; 628), 489 (362); Halmos, P. R., 808 (667); Halperin, I., 204 (71); Hamilton, H. J., 195 (44; 776); Harrold, O. G., 196 (44; 776), 196 (44; 776), 207 (72), 498 (362), 807 (668); Hartman, P., 340 (229), 490 (362), 807 (668); Hedge, L. B., 205 (88); Hedlund, G. A., 206 (79); Heins, A. E., 207 (72), 494 (363); Heins, M. H., 207 (44; 787 ), 496 (363); Helmer, O., 494 (363), 807 (668); Hestenes, M. R., 207 (72), 207 (72); 494 (519), 496 (363); Hille, E., 341 (88), 489 (364); Hoel, P. G., 490 (364); Hollcroft, T. R., 48 (44; 773), 207 (72), 806 (668); Hopkins, C., 205 (73), 341 (229); Hyers, D. H., $196(44 ; 777), 805$ (669), Huntington, E. V., 490 (364).

Ingraham, M. H., 495 (364); Isaacs, R. P., 340 (89).

Jackson, D., 206 (73), 494 (364), 806 (669); Jacobson, N., 206 (89), 341 (230), 489 (235), (835); James, R. D., 196 (44; 776); Jennings, S. A., (835); John, F., 204 (73), 807 (669); Johnson, M. M., 495 (365); Jones, B. W., 205 (73); Jones, F. B., 206 (44; 788), $207(44 ; 787)$.

Kac, M., 340 (230), 342 (365); van Kampen, E. R., 342 (365); Kaplan, W., 806 (669); Kasner, E., 48 (44; 640), 340 (230), 491 (365), 810 (669), (830); Kazarinoff, D. K., 194 (44; 782); Kelley, J. L., 206 (44; 788), 207 (74), 489 (365), 808 (667); Kempner, A. J., 206 (74); Kennedy, E. S., (835); Kenney, J. F., 194 (44; 782); Kershner, R. B., 495 (366), 807 (668); Kimball, W. S., 808 (670); Knebelman, M. S., 809 (670); Koehler, F., 494 (366); Kolchin, E. R., 340 (230), 808 (521); Koopman, B. O., 341 (89); Korzybski, A., 205 (74), (836); Kossack, C. F., 496 (366).

Laderman, J., (660); Lamoreau, F. L., 205 (44; 641); Lamson, K. W., 49 (44; 641); Lancaster, O. E., 204 (75); Lanczos, C., 194 (44; 783); Langer, R. E., 806 (670); Larguier, E. H., 207 (75), 341 (89); Latimer, C. G., 493 (366), 807 (671); Lay, Y., 493 (367); Lehmer, D. H., 342 (235), 807 (671); Leighton, W., 208 (44; 788), 809 (660); Levinson, N., 208 (44; 788), 208 (44; 789), 491 (236); Levitzki, J., 810 (671); Lewis, D. C., 809 (671); Lonseth, A. T., 497 (367); Lorch, E. R., 809 (519), (830); Lowan, A. N., 49 (44; 641), 49 (44; 641), 49 (44; 774), 49 (44; 774), 491 (367), (660), (660); Lubben, R. G., 206 (75).

McCoy, N. H., 808 (672); McDaniel, W. C., 494 (368); McEwen, W. H., 208 (76); McMillan, B., 340 (234); McShane, E. J., 205 (76), 208 (76), 208 (76), 489 (369), 491 (369), 806 (673), 810 (672), 810 (673), 810 (673), 810 (673); MacColl, L. A., 48 (44; 642); MacLane, S., 194 (44; 783), 205 (77), 208 (77), 340 (231), 491 (367), (662), (831); MacNeille, H. M., 205 (77); Maddaus, I., 808 (672); Maker, 
P. T., 491 (368); Malkin, I., 810 (672); Mancill, J. D., 49 (44; 774); Manning, D., 48 (44; 642); Manning, W. A., 205 (78); March, H. W., 494 (368); Maria, A. J., (835); Martin, M. H., 205 (78); Martin, V., 208 (78); Martin, W. T., 489 (358), 807 (663), (836); Mehlenbacher, L. E., 806 (522); Menger, K., 48 (44; 785); Mewborn, A. B., 497 (369), 498 (520); Michal, A. D., 196 (44; 777), 196 (44; 777), 497 (369), 498 (520), 811 (674), 811 (674), 811 (674); Milgram, A. N., 495 (520); Miller, E. W., 811 (829); Miller, H. C., 341 (231), 498 (369), 498 (370); Montgomery, D., 808 (674), 810 (674), (830), (836); Moore, C. N., 206 (79); Moore, M. G., 494 (370); Morrey, C. B., 497 (370), 810 (675); Morse, M., 206 (79), 489 (370); Moskovitz, D., 805 (675); Muhly, H. T., 489 (371), 807 (675); Murdoch, D. C., 340 (231), (837); Musselman, J. R., 194 (44; 783).

Nakayama, T., 340 (231); Nesbitt, C. J., $194(44 ; 783)$; Neuhaus, A., 808 (675); von Neumann, J., 205 (79); Niven, I. M., 490 (371).

Oakley, C. O., 204 (80); Oberg, E. N., 806 (676); O'Connor, R. E., 495 (236); Oldenburger, R., 194 (44; 784), 208 (80), 489 (371), 808 (676), (837); Ollmann, L. F., 495 (236), 807 (676); Opatowski, I., 806 (676); Ore, O., 208 (80), 340 (231), 340 (232), 809 (520); Oxtoby, J. C., 206 (80).

Parker, W. V., 495 (355); Paydon, J. F., 808 (681); Peebles, G. H., 49 (44; 785), 494 (372), 806 (677); Perlin, I. E., 808 (677); Pettis, B. J., 206 (88), 811 (677), 811 (677); Phillips, R. S., 494 (372), 495 (372); Pinney, E., 196 (80); Pitcher, E., 194 (44; 784), 205 (80), 806 (677), 810 (678); Prenowitz, W., (827); Price, G. B., 204 (81); Puckett, W. T., 208 (81), 490 (372), 809 (678).

Radó, T., 490 (373), 495 (373), 495 (373); Rainville, E. D., 194 (44; 784), 494 (373), 495 (374), 495 (374); Randels, W. C., 490 (374); Randolph, J. F., (837); Raudenbush, H. W., 809 (679); Reade, M., 207 (85), 494 (357); Rees, C. J., (837); Reichelderfer, P. V., 495 (373); Reid, W. T., 207 (72), 208 (81), 494 (374), 807 (679), 810 (678); Riblet, H. J., 339 (232), (838); Rinehart, R. F., 194 (81); Ritt, J. F., 491 (236), 808 (520), 808 (521), 809 (679); Roberts, J. H., 490 (374), (838); Robertson, M. S., 48 (44; 786), 491 (375), 810 (679); Robinson, C. V., (832); Robinson, L. B., 208 (90); Robinson, R. M., 195 (44; 644), 498 (375); Rodabaugh, L. D., 490 (521); Rosenbaum, B., (838); Ross, A. E., 807 (679); Rosser, B., 339 (90), 341 (90), 809 (523); Roth, W. E., 808 (679); Rothe, E. H., 194 (44; 785).

Schaeffer, A. C., 493 (234), 495 (237), 808 (680); Schilling, O. F. G., 205 (44; 789), 208 (77), 340 (231), 491 (375), (523); Schneckenburger, E. R., 807 (680); Schoenberg, I. J., 205 (79), 810 (680); Schwartz, A., (680); Schwartz, H. M., 491 (375); Schweigert, G. E., 204 (82), 495 (376); Schweitzer, A. R., 208 (44; 789), (681), (681); Scott, W. T., 205 (82), 494 (376), 807 (521), 809 (523), (838); Seward, D. M., 490 (376); Sewell, W. E., 491 (237), 491 (237), (523), (523), (524); Shaw, A. A., 195 (44; 777), 196 (44; 777); Shohat, J. A., 49 (44; 774), 205 (82), 208 (82), 489 (376), 490 (377); Sigley, D. T., (839); Simmons, H. A., 808 (681); Sinkov, A., 340 (232); Smiley, M. F., 805 (521); Smith, F. C., 204 (82); Sobczyk, A., 805 (682); Sorgenfrey, R. H., 341 (83); Spencer, V. E., 340 (90), 490 (377); Steenrod, N. E., 807 (682); Sternberg, W., (682); Stoker, J. J., 205 (70); Struik, R. R., 205 (83); Sugar, A., 206 (83); Swain, R. L., 341 (91); Swingle, P. M., 498 (377); Szász, O., 205 (83), 494 (356), 495 (377); Szegö, G., 811 (831).

Tamarkin, J. D., 205 (82); Taylor, A. E., 195 (44; 778), 196 (44; 778), 196 (44; 778); Thrall, R. M., 493 (377), 806 (683); Thurman, G. R., 204 (68); Tompkins, C. B., 489 (370); Torrance, C. C., 206 (83); Trimble, H. C., 493 (237); Trjitzinsky, W. J., 206 (44; 790), 808 (683), (839); Tucker, A. W., (839).

Ulam, S. M., 48 (44; 786), 206 (83), 340 (232). 
Valentine, F. A., 195 (44; 778); Vance, E. P., 495 (237); Vassell, A., 341 (91); Vaughan, H. E., 495 (377); Vickery, C. W., 491 (378), 491 (378), 809 (683), 809 (683), 810 (683), 810 (684).

Wakerling, R. K., 497 (378); Wald, A., 340 (232), 340 (238); Wall, H. S., 205 (82), 494 (376), 807 (521), 809 (523), (838); Wallace, A. D., 48 (44; 773), 206 (84), 208 (84), 341 (233), 341 (233), 490 (379), 491 (378), 491 (521), 807 (684); Walsh, J. L., 208 (84), 208 (84), 341 (233), 491 (379); Walton, L. F., 497 (379); Ward, J. A., 493 (380); Ward, M., 341 (86), 341 (91), 341 (92), 342 (92), 491 (91), 491 (92), 491 (92), 495 (91), 495 (92), 808 (522), 809 (524); Warschawski, S. E., 206 (85), 489 (361); van Waters, M., 205 (83); Webster, M. S., 806 (684); Weisner, L., 49 (44; 643), 49 (44; 775); Weyl, F. J., 495 (380); Whaples, G. W., 806 (684); Wheeler, A. H., (839); Whitman, P. M., 806 (685); Whitney, H., 342 (233); Whyburn, G. T., 206 (85), 208 (85), 490 (380), 807 (522), 807 (829), 809 (685), 809 (685); Wiener, N., 204 (69), 340 (234); Wilcox, L. R., 495 (380), 806 (685); Wolf, M. C., 808 (686); Wolfowitz, J., 340 (232); Wyman, M., 196 (44; 779), 497 (380), 498 (381), 811 (674).

Youngs, J. W. T., 490 (373), 810 (686), 810 (686).

Zippin, L., 808 (674), 810 (674), (830), (836); Zorn, M., 342 (238), 497 (381).

Personal Notes:

Adams, C. R., 63, 199, 202, 203, 338, 339, 487, 641, 804; Adkisson, V. W., 656; Agnew, R. P., 338, 802; Ahlfors, L. V., 349; Alaoglu, L., 350, 515; Albert, A. A., 202, 802, 805, 826; Albert, G. E., 350; Albert, R., 514; Allendoerfer, C. B., 656; Ambrose, W., 65, 828; Amelotti, E., 66; Ames, V. A., 350; Archibald, H. F., 656; Archibald, R. C., 2, 3, 5, 6, 22, 27, 30, 198, 202; Archibald, R. G., 201, 202; Arnold, H. A., 828; Artin, E., 488, 655, 656; Ayres, F., 350; Ayres, W. L., 30, 339, 488.

Baillie, D. C., 64; Bakst, A., 30; Bargmann, V., 828; Barkas, W. H., 828; Barnes, J. L., 656; Barnett, I. A., 492; Barnett, W. A., 64; Barnum, H. H., 658; Barone, H. G., 350; Bartels, R. C. F., 350; Basoco, M. A., 63; Behmann, H., 63; Bell, E. T., 5, 7, 8, 195, 488, 802; Bell, P. O., 656; Bellman, R., 514; Benac, T. J., 66; Bergmann, P. G., 828; Berkeley, E. C., 22; Berlin, T., 514; Bernet, E. J., 350; Bernstein, B. A., 63, 64; Berry, E. M., 64; Besancon, R. M., 65; Betz, E. E., 658; Birkhoff, G., 7, 8, 349, 828; Birkhoff, Mrs. G., 7; Birkhoff, G. D., 5, 7, 22, 28, 30, 198, 201, 203, 353, 487, 515, 641, 655, 656, 804, 826; Birnbaum, W. Z., 657; Blaschke, W., 655; Blichfeldt, H. F., 349, 497; Bliss, G. A., 5, 7, 30, 63, 201, 225, 339; Bliss, Mrs. G. A., 7; Blumenthal, L. M., 655, 657; Boas, R. P., 658; Bochner, S., 63, 349, 657; Bohnenblust, H. F., 657; Bohr, N., 63; Boldyreff, A. W., 64; Bompiani, E., 515; Boon, F. C., 658; Borel, E., 655; Bouligand, G., 226; Bowden, J., 656; Bowley, A. L., 655; Boyce, F. W., 350; Boys, C. V., 515; Brady, C. P., 350; Brahana, H. R., 63; Brauer, A. T., 828; Brauer, R., 493; Bray, H. E., 64, 198, 202; Breiland, J., 66; Brewer, B. W., 66, 350; Bricard, R., 225; Bridgman, P. W., 655; Briggs, L. J., 350; Brixey, J. C., 827; Brown, D. M., 350; Brown, E. W., 22, 338, 343; Browne, E. T., 338, 339; Bryan, J. S., 199; Buchanan, D., 515; Buchanan, H. E., 63, 802; Burgatti, P., 66; Burington, R. S., 193; Burniat, P., 514; Burrell, E. L., 227; Bush, V., 349; Bushey, J. H., 515; Bussey, W. H., 30; Butler, N. M., 4, 5, 20; Butler, Mrs. N. M., 4; Byrd, N. P., 514.

Cairns, W. D., 193, 198, 514, 657, 803; Calkin, J. W., 64; Campbell, A. D., 47, 338, 488, 828; Campbell, G. A., 515; Campbell, Mrs. H. E. O., 8; Carson, J. R., 515; Cartan, E., 225, 515, 655; Carver, W.B., 199, 339; Cassity, C. R., 350; Chit- 
tenden, E. W., 339, 493; Church, A., 202, 349, 657; Cinquine, S., 515; Clark, C. E., 350, 658; Coble, A. B., 30, 63, 201, 203, 487, 641; Cochran, W. G., 63; Cohen, T., 226; Cole, M. B., 354; Collins, O. C., 657; Conway, A., 349; Coolidge, J. L., 2, 30, 199, 226; Cope, T. F., 657; Coral, M., 225; Cordrey, W. A., 827; Courant, R., 349; Coxeter, H. S. M., 802; Cowgill, A. P., 657; Cremer, H., 63; Curtiss, A. J., 7 ; Curtiss, D. R., 5, 7, 22, 198, 516.

Dale, S. S., 6; Daly, J. F., 5; van Dantzig, D., 655; Davis, H. T., 349; Day, M. M., 828; Dearborn, D. C., 657; DeCicco, J. J., 350; DeCou, E. E., 655; Delens, P., 225; Denjoy, A., 225, 226, 515; Dennis, F. L., 66, 350; Dickson, L. E., 2, 30, 492, 493, 516, 802; Dickstein, S., 4; Dietzold, R. L., 30; Dieudonné, J., 225; Dines, L. L., 804; Dirac, P. A. M., 349; Dobbie, J. M., 351; Doob, J. L., 63; Douglas, J., 225; Dowker, C. H., 64, 351, 828; Doyle, T. C., 658; Doyle, W. C., 351; Dresch, F. W., 354; Dresden, A., 30, 339, 487; Dumas, S., 227; Duncan, D. C., 65; Dunford, N., 63, 64; Dunkel, O., 656.

Eachus, J. J., 658; Eaton, Mrs. D. R., 30; Eaton, J. E., 828; Eckert, W. J., 6; Edmondson, T. W., 66; Eiesland, J. A., 349; Eisenhart, L. P., 5, 7, 22, 23, 26, 30, 63, 201, 339; Elston, J. S., 22 ; Emch, A., 22 ; Erdös, P., 828; Evans, G. C., 5, 22, 201, 202, 338, 802, 803, 804; Evans, H. P., 64; Ewing, G. M., 827; Eyraud, 827.

Fabry, L., 354; Fancher, M. E., 514; Favard, J., 225; Fermi, E., 63, 226, 826; Ferry, F. C., 7, 8, 15; Few, W. P., 488; Feynman, R. P., 514; Fialkow, A., 227; Ficken, F. A., 65, 351; Findlay, W., 349; Fischer, E., 63; Fisher, R. A., 225; Fiske, N. P., 7; Fiske, T. S., 2, 4, 5, 6, 7, 8, 12, 30, 203; Fite, W. B., 3, 4, 29, 30, 201, 202, 339, 805; Fite, Mrs. W. B., 4; Flexner, W. W., 828; Fock, W., 655; Follin, J. W., 514; Ford, L. R., 193, 199, 804; Ford, W. B., 227; Forsyth, C. H., 516; Fort, T., 2, 202, 805; Fowler, M. C., 351; Fox, R. H., 828; Frame, J. S., 64; Franklin, F., 227; Franklin, P., 22, 23, 339; Frick, B. M., 30; Frink, O., 487; Fry, T. C., 4, 22, 29, 30, 203, 641, 803, 804; Fubini, G., 828; Fuller, G., 64; Furtwängler, P., 659.

Gallatin, A. R., 354; Galvin, C. F., 351; Garbe, E. R., 66; Gatewood, B. E., 827; George, T. C., 65; Gere, B. H., 351; Gergen, J. J., 198; Germond, H. H., 64; Ghent, K. S., 657; Gibson, A. H., 349; Gill, B. P., 30, 202; Gödel, K., 828; Goldstein, S., 350; Goldsworthy, E. C., 353; Grable, E. S., 66; Graesser, R. F., 657; Graffi, D., 63; Graustein, W. C., 202, 339, 803, 804; Graves, C. H., 351, 827; Graves, L. M., 63, 225, 516, 805; Green, J. W., 351, 828; Greenebaum, M., 351; Greenwood, R. E., 66; Greer, E., 65; Griffin, F. L., 493; Gropper, L., 354; Grove, V. G., 193; Guinand, A. P., 655.

Haberzetle, M. B., 351; Hailperin, T., 828; Hall, D. W., 351, 658; Hall, N. A., 351; Halmos, P. R., 351, 828; Halperin, I., 63, 657; Hammer, P. C., 351; Hanson, W. R., 66; Hardy, G. H., 655; Harkin, D. C., 227; Harrell, E. G., 64; Harris, E., 66; Harrold, O. G., 656; Hart, W. L., 339; Hartman, P., 351; Havens, R. J., 351; Hayden, C., 65; Hedge, L. B., 658; Hedlund, G. A., 47, 63, 227; Hedrick, E. R., 2, 5, 7, 30, 195, 201, 656; Hedrick, Mrs. E. R., 195; Heins, M. H., 658; Helmer, O., 65; Hemraj, P., 354; Henderson, R., 22, 202; Hendrickson, M., 658; Herzberger, M., 63; Herzog, F., 658; Hestenes, M. R., 225; Higdon, R. A., 657; Hilbert, D., 226; Hildebrandt, T. H., 201, 339; Hille, E., 804; Hillman, A., 514; Hobbs, A. W., 227; Hoel, P. G., 828; Hollcroft, T. R., 30, 202; Hopper, G. M., 657; Hostetter, I. M., 657; Hotelling, H., 349, 826; Hsü, H.-Y., 351; Huber, A., 656; Hughes, H. M., 66; Huntington, E. V., 63, 201.

Ingham, A. E., 826, 828; Ingraham, M. H., 2, 30, 63, 492, 804.

Jackson, D., 2, 7, 8, 30, 339, 493; Jackson, R. F., 65; James, R. D., 63, 195, 
657; Jeans, J., 226; Jessop, C. M., 516; Jewett, F. B., 226; Joffe, S. A., 2; Johannes, K., 354; Johanson, R. N., 516; Jones, G. E., 353; Jones, H., 30.

Kac, M., 828; Kagan, B., 655; Kaltenborn, H. S., 827; Kaplan, E. L., 514; Karapetoff, V., 22, 827; von Kármán, T., 203, 339; Kasner, E., 2; Keeler, I. F., 351; Kempner, A. J., 4 ; Kennedy, E. S., 658; Keston, J. F., 354; Kincaid, W. M., 514; King, W. L. M., 8, 11; Kleene, S. C., 828; Kline, J. R., 30, 63, 198, 201, 338, 804, 805; Klobou ček, J., 659; Koehler, F., 658; Koopman, B. O., 63, 349; Kossack, C. F., 658; Krall, H. L., 227; Krause, E. H., 351; Kroon, R. P., 22; Krull, W., 226; Kuratowski, C., 4.

Ladue, P., 4; Landers, A. W., 227; Landry, A. E., 804; Lang, G. B., 658; Langer, R. E., 4, 6, 18, 802; Larew, G. A., 197; Latimer, C. G., 63, 492; Lax, B., 514; Lebesgue, H., 655; Lefschetz, S., 5, 7, 30, 226; Lefschetz, Mrs. S., 7; Lehmer, D. H., 802; Lehmer, D. N., 209; Lengyel, B. A., 658; Leray, J., 226; Lester, C. A., 657; Leutner, W. G., 193; Levin, M., 354; Levine, J., 827; Levy, H., 63; Lewis, D. C., 63, 827; Lewy, H., 63; Lieber, H. G., 65; Lieber, L. R., 65; von Lindemann, F., 354; Ling, G. H., 657; Lippmann, C. W., 514; Loney, S. L., 659; Longley, W. R., 202, 338; Lorch, E. R., 30, 201; Lowan, A. N., 350.

McCoy, N. H., 63, 198; McDaniel, R. R., 352; McDaniel, W. C., 827; McShane, E. J., 5, 197, 202, 225, 339, 349, 802, 805; MacDuffee, C. C., 193, 493, 802, 805; MacLane, S., 488; MacNeille, H. M., 488; Maddaus, I., 65; Madow, W. G., 352; Maker, P. T., 352; Mandelbrojt, S., 225; Manià, B., 515; Manning, D., 658; Manson, S., 514; Margenau, H., 828; Maria, A. J., 354; Marshall, W., 515; Martin, M. H., 658; Martin, W. T., 339, 805; Marty, F., 225; Mason, T. E., 659; Massey, H. S. W., 64; Mauch, M. E., 352; May, L. B., 66; Meder, A. E., 3, 30, 201, 202; Meissner, E., 659; Menger, K., 63, 225, 514, 655; Merriman, G. M., 65; Michal, A. D., 22, 29, 339, 349, 497, 658; Milgram, A. N., 655; Mintzer, H., 514; Moisseiff, L. S., 22; Montague, H. F., 658; Montel, P., 655; Mooney, R. L., 65; Moore, C. N., 805; Moore, L. T., 227; Moore, M. G., 352; Moore, R. L., 4, 5, 7, 8, 30, 198, 199, 200; Moran, C., 66; Morrey, C. B., 65, 805; Morris, R., 2; Morse, M., 22, 29, 203, 225, 226, 641, 655, 804; Moursund, A. F., 658; Muehlmann, P., 827; Müller, M., 656; Müller, R., 516; Mullins, G. W., 30, 202; Murnaghan, F. D., $63,199,804$.

Nelson, A. L., 193; Netzorg, D. L., 66, 352; Neugebauer, O., 227, 642; von Neumann, J., 63, 199; Newman, M. H. A., 515; Newsom, C. V., 804; Newton, A. V., 65; Neyman, J., 65; Nicholson, T., 227; Nierenberg, W., 514; Nilson, E. N., 658; Niven, I. M., 352; Norris, M. J., 514; Northcott, D. G., 655.

d'Ocagne, M., 66; Oldenburger, R., 658; Ollivier, A., 516; Ollmann, L. F., 828; O'Quinn, R. L., 516; Ore, O., 805; Ormondroyd, J., 22; Osgood, W. F., 802; Ostrander, J. E., 66.

Parker, W. V., 227; Pearl, R., 349; Peebles, G. H., 352; Pegram, G. B., 22, 24 ; Pepper, P. M., 655; Pepperitz, E., 66; Perez, Mrs. R. F., 7; Perkins, D. T., 352, 515; Perlis, S., 352; Peschl, E., 656; Peterson, J. K., 65; Peterson, T. S., 66, 658; Pettis, B. J., 515; Pflüger, A., 656; Phillips, E. C., 65; Phillips, H. B., 22, 23, 339; Phillips, R. S., 828; Picard, E., 226; Picone, M., 655; Pierce, J. A., 352 ; Pierpont, J., 2, 30, 66, 481; Pirenian, Z. M., 65; Pitcher, E., 658; Plass, Mrs. M. H., 65; Plimpton, G. A., 6; Poor, V. C., 227; Predella, P., 659; Price, IG. B., 658; Primakoff, H., 352; Pupin, M. I., 2; Putnam, R. G., 30; Putnam, T. M., 30.

Quade, W., 64.

Rademacher, H. A., 63, 199, 349; Rado, T., 225, 802; Rainich, G. Y., 63; Randolph, J. F., 63; Rees, M. S., 30; Rees, P. K., 827; Reeve, W. D., 22, 30; Reich- 
elderfer, P. V., 658; Reid, W. T., 225; Reingold, H., 65, 352; Reinsch, B. P., 226; Reissner, E., 352; Rempfer, R. W., 352; Riabouchinsky, D., 22; Riblet, H. J., 658; Richardson, R. G. D., 2, 30, 199, 202, 805; Rider, P. R., 658; Rietz, H. L., 22; Rietz, J. C., 516; Risselman, W. C., 659; Ritt, J. F., 5, 47, 338; Robbins, H. E., 352, 658; Robertson, H. P., 202, 349; Rodabaugh, L. D., 352; Roger, F., 225; Roosevelt, F. D., 8, 10; Ross, A. E., 658; Rosser, B., 827; Rothe, R., 515; Rozet, F., 514; Russell, H. N., 349, 515; Rutt, N. E., 227.

Sadowsky, M., 655; Saslaw, S. S., 65, 352; Schaaf, S. A., 514; Schaeffer, A. C., 658; Schilling, O. F. G., 516; Schmeidler, W., 656; Schmieden, K., 64; Schnirelmann, L. G., 66; Schoenberg, I. J., 655; Schotten, H., 659; Schouten, J. A., 655; Schreiber, T. S., 514; Schwartz, A., 828; Schweigert, G. E., 227, 828; Scott, W. T., 352, 516, 828; Seifert, H., 226; Sellery, G. C., 803; Severi, F., 515; Shapley, H., 226, 349; Shaub, H. C., 658; Sheffer, H. M., 658; Sherman, B., 514; Shiffman, M., 352; Shreve, D. R., 353; Sierpiński, W., 655; Signorini, A., 515; Simon, W. G., 193, 201; Simond, R. G., 353; Singleton, H. E., 514; Sisk, A., 65; Sklaar, G. J., 7, 30; Smiley, M. F., 225; Smith, A. H., 658; Smith, C. E., 30; Smith, D. E., 3, 6, 22, 29, 30; Smith, H. L., 828; Smith, I. W., 656; Smith, P. A., 9, 30, 828; Snyder, V., 3, 5, 7, 22, 29, 30, 339, 802; Snyder, Mrs. V., 7; Snyder, W. S., 658; Sokolnikoff, I. S., 492; Sommerfeld, A., 655; Soodak, H., 514; Spampinato, N., 226; Sperry, P., 65; Springer, C. E., 353; Stabler, E. L., 7; Stabler, Mrs. E. L., 7 ; Staley, R. C., 353; Steenrod, N. E., 516; Stephany, E., 65; Stephens, R. C., 827, 828; Stetson, J. M., 197; Stone, M. H., 198, 202, 337, 802, 805; Stopher, E. C., 827; Stouffer, E. B., 803; Struik, D. J., 22, 339; Synge, J. L., 5, 22 ; Szász, O., 349; Szegö, G., 195, 349, 497, 805, 826; Szegö, Mrs. G., 497.

Tamarkin, J. D., 63, 642; Tate, E. L., 514; Teles, M., 353; Thomas, J. M., 349, 487; Thomas, T. Y., 5, 195, 339, 497; Thompson, A. R., 30; Threlfall, W., 656; Timerding, H., 64; Timoshenko, S., 655; Tonelli, L., 515; Topel, B. J., 655; Tornheim, L., 66, 353, 354; Trimble, H. C., 349; Tripp, M. O., 65; Trjitzinsky, W. J., 349; Tukey, J. W., 658; Turing, A. M., 353; Turner, M. J., 66; Turrittin, H. L., 827; Tzitzéica, G., 659.

Uhlenbeck, G. E., 353; Ulam, S. M., 63; Ulrich, F. E., 227, 353; Updegraff, M., 66; Urbain, G., 516.

Vance, E. P., 828; Van de Carr, J. C., 66; Van Stockum, W. J., 354; Van Vleck, J. H., 515; Veblen, O., 2, 22, 63, 488, 641, 656, 803, 804; Vehse, C. H., 353; Vest, M. L., 66; Vezeau, W. A., 828; Vincenzini, P., 656.

Wade, T. L., 827; Wadsworth, G. P., 353; Wagner, P. S., 227; Wagner, R. W., 658; Waidelich, D. L., 65; Wakerling, R. K., 828; Wald, A., 828; Walker, G. L., 65; Wallman, H., 828; Walsh, J. L., 201, 339; Ward, J. A., 828; Ward, M., 63; Watson, G. N., 826; Weaver, W., 7, 198, 641, 804; Weaver, Mrs. W., 7; Weber, W., 64; Wehausen, J. V., 353, Weiss, M., 339; Welker, E. L., 353; deWet, J. S., 828; Weyl, H., 202, 349, 487, 656; Wheeler, A. P., 339, 802; Wheeler, C. H., 197, 201; White, H. S., 2, 5, 7, 30; Whitehead, G. W., 354; Whitney, H., 349; Whyburn, G.T., 198, 199, 203, 226, 641, 805; Whyburn, W. M., 226, 497; Wickenden, W., 193; Wiener, N., 5, 22, 487; Wilbur, W. E., 354; Wilder, R. L., 5, 63, 202; Wilks, S. S., 63; Williams, K. P., 339; Williams, W. L., 65; Williamson, J., 22; Wilson, A. H., 516; Wilson, W. A., 63; Winkelmann, M., 64; Winsor, C. P., 65; Wintner, A., 63, 349; Wishard, A., 353; Wolf, L. A., 353; Wood, F., 65; Wood, H. A., 353; Wood, R. W., 226; Wrench, J. W., 353.

Yanney, B. F., 201.

Zariski, O., 349, 515. 
Prizes and Medals:

Albert $I^{\text {er }}$ de Monaco Prize, 225; Henri Becquerel Foundation Prize, 225; Bôcher Prize, 199; Bruce Medal, 349; Chauvenet Prize, 226; Cole Prize in Algebra, 203; Copley Medal, 63; Elliott Cresson Medal, 515; François Deruyts Prize, 514; James Alfred Ewing Medal, 349; Francoeur Prize, 225; 1939 John Fritz Gold Medal, 226; Girbal-Baral Foundation Award, 225; Grand Prize for Mathematical Sciences, 225; John L. Lewis Prize, 515; Lobatchewsky Prize, 225; Lorentz Medal, 655; Malaxa Prize, 226; Marquet Prize, 225; Mittag-Leffler Institute Medal, 226; Nobel Prize in Physics, 63; Order of Merit, 226; Poncelet Prize, 225; William Lowell Putnam Mathematical Prize, 514; Research Corporation of New York Prize, 349; Royal Society Medal, 225; Rumford Medal, 226; James Scott Prize, 349.

Universities and Technical Schools:

Bell Telephone Laboratories, 29; Brooklyn College, 9, 514; Brown University, 642; University of California (Berkeley), 514; Catholic University of America, 804; University of Chicago, 225; University of Cincinnati, 63; College of the City of New York, 9, 514; Columbia University, 4, 6, 9; Cooper Union, 201, 514; Duke University, 338; Franklin Institute, 515; Georgetown University, 804; Harvard University, 514, 826; Institute for Advanced Study, 63; Iowa State College, 514; Kent State University, 201; Massachusetts Institute of Technology, 514; Mathematical Institute of Kazan, 225; Mississippi Woman's College, 514; Mittag-Leffler Institute, 26, 226; Mount Union College, 201; University of New Mexico, 804; New York University, 9; University of Notre Dame, 514, 655; Queens College, 9; University at Sofia, 655; University of Toronto, 826; Tufts College, 201; West Point, 8, 30; Yale University, 63, 349, 826. 